\title{
Commissioning activities and first results from the collective Thomson scattering diagnostic on ASDEX Upgrade (invited)
}

Meo, Fernando; Bindslev, Henrik; Korsholm, Søren Bang; Furtula, Vedran; Leuterer, F.; Leipold, Frank; Michelsen, Poul; Nielsen, Stefan Kragh; Salewski, Mirko; Stober, J.

Total number of authors:

12

Published in:

Review of Scientific Instruments

Link to article, DOI:

$10.1063 / 1.2989140$

Publication date:

2008

Document Version

Publisher's PDF, also known as Version of record

Link back to DTU Orbit

Citation $(A P A)$ :

Meo, F., Bindslev, H., Korsholm, S. B., Furtula, V., Leuterer, F., Leipold, F., Michelsen, P., Nielsen, S. K., Salewski, M., Stober, J., Wagner, D., \& Woskov, P. (2008). Commissioning activities and first results from the collective Thomson scattering diagnostic on ASDEX Upgrade (invited). Review of Scientific Instruments, 79(10), 10E501. https://doi.org/10.1063/1.2989140

\section{General rights}

Copyright and moral rights for the publications made accessible in the public portal are retained by the authors and/or other copyright owners and it is a condition of accessing publications that users recognise and abide by the legal requirements associated with these rights.

- Users may download and print one copy of any publication from the public portal for the purpose of private study or research.

- You may not further distribute the material or use it for any profit-making activity or commercial gain

- You may freely distribute the URL identifying the publication in the public portal 


\title{
Commissioning activities and first results from the collective Thomson scattering diagnostic on ASDEX Upgrade (invited) ${ }^{a)}$
}

\author{
F. Meo, ${ }^{1}$ H. Bindslev, ${ }^{1}$ S. B. Korsholm,${ }^{1}$ V. Furtula, ${ }^{1}$ F. Leuterer ${ }^{3}$ F. Leipold, ${ }^{1}$ \\ P. K. Michelsen, ${ }^{1}$ S. K. Nielsen, ${ }^{1}$ M. Salewski, ${ }^{1}$ J. Stober, ${ }^{2}$ D. Wagner, ${ }^{2}$ and P. Woskov ${ }^{3}$ \\ ${ }^{1}$ Association EURATOM, Ris $\phi$ National Laboratory for Sustainable Energy, Technical University of Denmark, \\ DK-4000 Roskilde, Denmark \\ ${ }^{2}$ Max-Planck-Institut für Plasmaphysik, EURATOM Association, D-85748 Garching, Germany \\ ${ }^{3}$ MIT PSFC, Cambridge, Massachusetts 02139, USA
}

(Presented 12 May 2008; received 12 May 2008; accepted 11 August 2008; published online 31 October 2008)

\begin{abstract}
The collective Thomson scattering (CTS) diagnostic installed on ASDEX Upgrade uses millimeter waves generated by the newly installed $1 \mathrm{MW}$ dual frequency gyrotron as probing radiation at 105 GHz. It measures backscattered radiation with a heterodyne receiver having 50 channels (between 100 and $110 \mathrm{GHz}$ ) to resolve the one-dimensional velocity distribution of the confined fast ions. The steerable antennas will allow different scattering geometries to fully explore the anisotropic fast ion distributions at different spatial locations. This paper covers the capabilities and operational limits of the diagnostic. It then describes the commissioning activities carried out to date. These activities include gyrotron studies, transmission line alignment, and beam pattern measurements in the vacuum vessel. Overlap experiments in near perpendicular and near parallel have confirmed the successful alignment of the system. First results in near perpendicular of scattered spectra in a neutral beam injection (NBI) and ion cyclotron resonance heating (ICRH) plasma (minority hydrogen) on ASDEX Upgrade have shown evidence of ICRH heating phase of hydrogen. () 2008 American Institute of Physics. [DOI: 10.1063/1.2989140]
\end{abstract}

\section{INTRODUCTION}

Fundamentally, in collective Thomson scattering (CTS) an incident probing radiation scatters off ion driven collective fluctuations in a plasma. The received scattered radiation emanates from the region where the probe and receiver beam patterns overlap (the scattering volume). The scattering spectrum carries information about the distribution of ion velocity components projected along the fluctuation wave vector $k^{\delta}=k^{s}-k^{i}$ where $k^{s}$ and $k^{i}$ are the wave vectors of the received scattered radiation and the incident probing beam, respectively. Scattering (reradiation) is essentially off electrons. However, fast ion information can be inferred from the waves the fast ions leave in the electron distribution. However, at scale lengths larger than the Debye length $\lambda_{D}$, the electron wake is essentially like an electron hole covering the electron. This leads to destructive interference of the scattering from the electron and its perturbation. Therefore, for scales larger than the Debye length the dominant scattering comes from the coherent fluctuations in the plasma caused by the collective response of the electrons to the dynamics of the ions. The criterion for collective scattering is expressed using the Salpeter parameter: ${ }^{1} \alpha=\left(\lambda_{D} k^{\delta}\right)^{-1}>1$. The Salpeter criterion set limits to the scattering geometry as a function of the probe frequency. By using gyrotrons (millimeter waves) as the source, the choice of geometries is virtually unrestricted. ${ }^{2}$ Further literature on CTS can be found in Refs. $3-5$. The CTS diagnostic has been used to measure fast ion

\footnotetext{
anvited paper, published as part of the Proceedings of the 17th Topical Conference on High-Temperature Plasma Diagnostics, Albuquerque, New Mexico, May 2008.
}

populations on other machine such as $\mathrm{JET}^{6}$ and TEXTOR. ${ }^{7,8}$ The microwave based fast CTS diagnostic installed on ASDEX Upgrade (CTS-AUG) uses millimeter waves generated by the newly installed 1 MW dual frequency gyrotron as probing radiation at $105 \mathrm{GHz} .{ }^{9}$ This paper will include a brief overview of the hardware in Sec. II and the diagnostic capability in Sec. III. Section IV will cover the commissioning activities carried out which will describe the prerequisites and pitfalls of a microwave based CTS system. Preliminary scattering results are shown in Sec. V along with discussion and commissioning activities still to be completed are covered in Sec. VI.

\section{THE CTS AT ASDEX UPGRADE HARDWARE}

The hardware description in this section will expand on the previous description presented in Refs. 10 and 11. The CTS-AUG uses millimeter waves generated by the $1 \mathrm{MW}$ dual frequency gyrotron (Odyssey-2). ${ }^{12}$ The $105 \mathrm{GHz}$ frequency mode is used as the probing radiation where power up to $620 \mathrm{~kW}$ for $10 \mathrm{~s}$ has been attained. Near backscattered radiation is collected by neighboring electron cyclotron resonance heating $(\mathrm{ECRH})$ antenna located in the same port. The CTS-AUG uses the main portion of the ECRH No. 2 transmission line described in detail in Ref. 9. The ECRH antennae are steerable enabling the possibility of different scattering geometries. The scattered radiation is transmitted to the CTS receiver in the HE11 mode via a $70 \mathrm{~m}$ corrugated waveguide to the free space propagation portion- the matching optics unit (MOU) box located in the gyrotron hall. Figure 1 illustrates the locations of some of the MOU components for 


\section{ECRH operation}

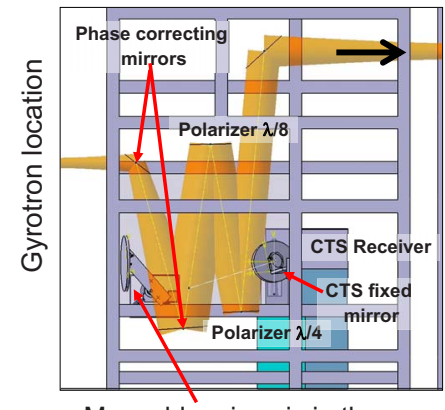

Moveable mirror is in the neutral position.
CTS operation

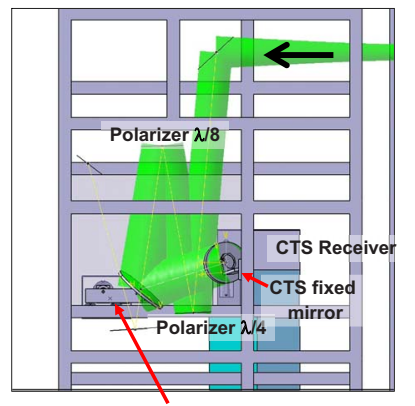

Moveable mirror is in the CTS operation mode.
FIG. 1. (Color online) Side view of the MOU No. 2 at ASDEX Upgrade. In gyrotron operation (left) the movable CTS mirror is positioned inward toward the gyrotron. In CTS measurement operation (right) the movable mirror is positioned inward to intercept the incoming radiation from the transmission line No. 2. A second fixed mirror directs radiation toward the CTS receiver horn (into the page).

the ECRH operation, namely, the phase correcting mirrors, two broadband polarizers, and the HE11 coupling mirror. Two additional mirrors for the CTS are installed in the same MOU. One is fixed to the MOU frame toward the side closer to the reader. The other is installed on a movable arm controlled by a differential step motor. Two modes of MOU operation are illustrated in Fig. 1 where during the "ECRH operation" mode, the movable mirror is in the outward position (left of Fig. 1) leaving an unobstructed beam path for the gyrotron power. In the "CTS operation," the movable mirror is rotated inward intercepting the incoming radiation between the second phase correcting mirror and the polarizer which redirects radiation to the fixed CTS mirror that couples radiation to the CTS receiver located on the other side of the MOU (into the page). Hence in this setup, the CTS system makes use of the two ECRH broadband polarizers for adjusting the beam polarization for optimum plasma propagation. The front end of the CTS receiver consists of a scalar corrugated horn, chopper mirror for liquid $\mathrm{N}_{2}$ calibration, and wire grid to counter the formation of standing waves. The radio frequency (rf) and intermediate frequency (IF) components are enclosed inside a metal box for electromagnetic compatibility shielding. The rf receiver components consist of two waveguide band-notch filters ${ }^{13}$ to suppress the gyrotron stray radiation. The other receiver components include an isolator, bandpass filters, and a broadband solid state voltage controlled variable attenuator (VCVA) capable of switching to $40 \mathrm{~dB}$ attenuation in $5 \mu \mathrm{s}$. VCVA is switched by the positive and negative slopes of the gyrotron gate pulse and is used to attenuate the radiation in the time window during the gyrotron beam voltage rise and fall where the radiation frequency chirps outside the notch filter bandwidth (see Fig. 3 in Sec. IV A) and where possible mode competition can occur causing spurious signal. The signal is downconverted to IFs by a broadband fundamental mixer driven by a $95 \mathrm{GHz}$ local oscillator. Since the power in the central part of the spectrum may be orders of magnitude larger than in the sideband, the IF frequencies are split by a triplexer into three bands: 4.0-9.0, 9.0-10, and 10-14 GHz. Hence the complete IF range of the receiver is between 4.0 and $14.0 \mathrm{GHz}$ that corresponds to rf's between about 100 and $110 \mathrm{GHz}$. Each band then goes through two stages of amplification $(\approx 30-40 \mathrm{~dB}$ gain each) by a low noise $(<1.5 \mathrm{~dB})$ and a high power IF amplifier pairs that are robust against gain compression. The first and second amplifiers have the $1 \mathrm{~dB}$ compression point at +15 and $+25 \mathrm{dBm}$, respectively. By the use of power dividers, the signal passes through a 50 channel filter bank having band widths of 100 $\mathrm{MHz}$ for the central channels and 500-1000 MHz for the outer ones (out of channel rejection at about $-30 \mathrm{~dB}$ ). The signals are then detected by Schottky diodes for further amplification by video amps. A series of 24 bit analog to digital synchronized detector cards digitize the signal (50 channels simultaneously) at $100 \mathrm{kHz}$.

\section{DIAGNOSTIC CAPABILITY OF CTS-AUG}

\section{A. Scattering geometries and pitch angle range}

The CTS-AUG provides measurements of the onedimensional fast ion velocity distribution projected along a direction which is defined by the scattering geometries. In particular, the scattering geometries may be varied to measure the distribution as a function projected on a wide range of pitch angles. Figure 2 shows two scattering geometries from near perpendicular to the magnetic field Fig. 2(a) to near parallel to the magnetic field $\angle\left(k^{\delta}, B\right) \approx 160^{\circ}$ which is the limit imposed by the steering capability of the ASDEX Upgrade ECRH antenna steering capability. The scattering geometry in Fig. 2 shows antenna steering setup in the counterion flow direction, hence the scattered spectra will be upshifted as opposed to the coion direction where $\angle\left(k^{\delta}, B\right)$ $\approx 20^{\circ}$ where the scattering spectra will be downshifted. The maximum limit of the parallel angles will also be, of course, depend on the spatial location of the scattering volume. For example, for a scattering volume located at $\rho_{\mathrm{pol}}=+(-) 0.6$ the positive (negative) sign indicates positions above (below) the magnetic axis will be limited to $\angle\left(k^{\delta}, B\right) \approx 120^{\circ}\left(165^{\circ}\right)$. The CTS diagnostic can, in principle, measure the bulk ion flow velocity both in the toroidal and poloidal directions.

\section{B. Electron Cyclotron Emission (ECE) background: Temporal and velocity resolutions}

The choice of scattering geometry determines to a large extent the spatial resolution, robustness of the beam overlap, and the resolving power. The resolving power is a measure of the accuracy with which the system can estimate the fast ion velocity distribution for a given velocity space resolution. ${ }^{14}$ The resolving power is directly proportional to beam overlap, the square root of the integration time and to the probe radiation power and inversely proportional to the background noise temperature $\left(T_{\text {receiver noise }}+T_{\mathrm{ECE}}\right)$. For the system at ASDEX Upgrade, the spectral range centered at $105 \mathrm{GHz}$, the central magnetic field is near $2.5 \mathrm{~T}$. This is the operating scenario chosen for CTS experiments where the electron cyclotron resonances are outside the plasma in order to minimize the ECE background. Residual emission is either due either to a high energy electron tail population, emitting into the spectral range via deeply relativistic Doppler shift, or due to a low temperature plasma outside the last 


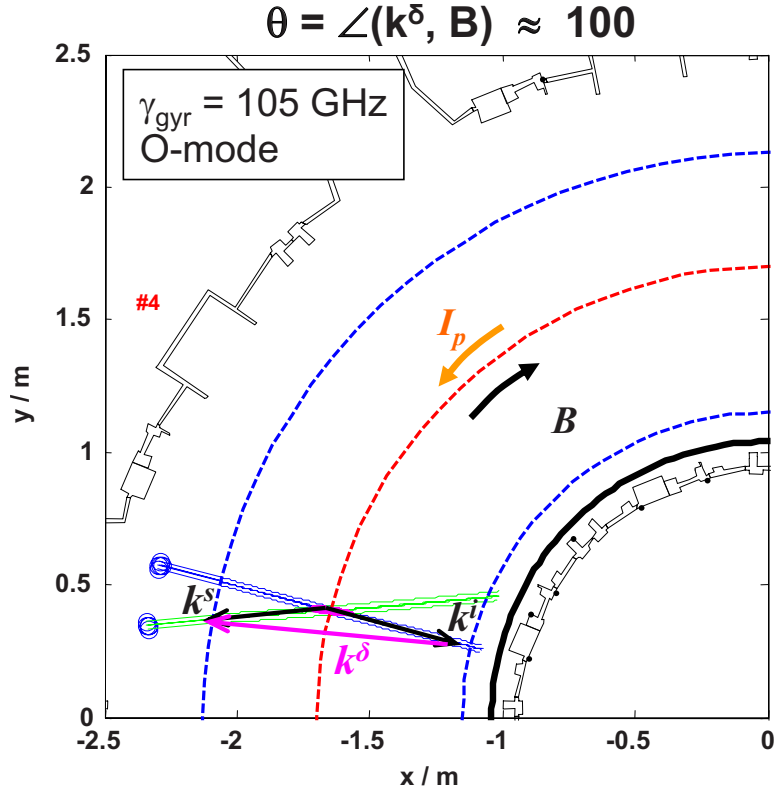

(a)

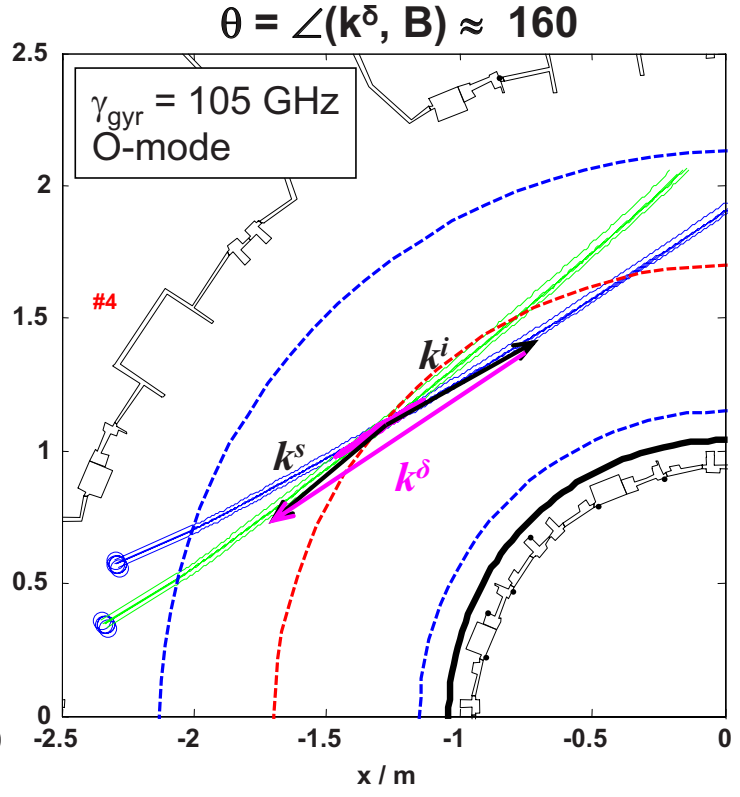

(b)

FIG. 2. (Color online) Top view of the CTS setup on ASDEX upgrade. The blue traces illustrate the probe beam emanating from antenna No. 1 while the green traces show the receiver beam to antenna No. 2. The radiation frequency is $105 \mathrm{GHz}$ in $O$-mode and the plasma scenario is such that the fundamental and first harmonic resonance are outside the plasma $\left(B_{o}=2.55 \mathrm{~T}, I_{p}=800 \mathrm{kA}\right)$ with a central electron density value of $6 \times 10^{19} \mathrm{~m}^{-3}$. The magenta ellipsoid is the scattering volume in the overlapping region defined by the intersection of the probe and receiver beams. Overlaid are the $k$ vectors $k^{i}, k^{s}$, and $k^{\delta}$ which are the incident, scattering, and fluctuation wave vectors, respectively. (a) is the scattering geometry for near perpendicular and (b) is for near parallel (maximum possible on ASDEX Upgrade for scattering volume in the center).

closed flux surface. Ray-tracing calculations assuming Maxwellian velocity distributions and a central electron temperature of $10 \mathrm{keV}$ have been carried out. Measurements have shown that the in-band ECE will generally be less than 500 eV. Except in very low-density discharges, we do not expect nonthermal tails on the electron velocity distributions in AUG. The expected scattering radiation is of few tens of eV ( $\mathrm{eV}$ being the radiated power); hence to discriminate the signal from noise and background we modulate the probe power. Integrating the signal over a finite time, $\tau$, and bandwidth, $\lambda_{B}$, the relative uncertainty in the estimate of the band and time-averaged spectral power density is the raw signal to noise ratio multiplied by $(\tau B)^{1 / 2}$. With bandwidths on the order of a gigahertz and integration times on the order of a millisecond this factor is on the order of 1000 yielding acceptable estimation accuracies. In the feasibility study in Ref. 15 uncertainties of the expected CTS spectra were estimated with the use of additional information obtained from other diagnostics. Finite uncertainties in these sets of information (nuisance parameters) also contribute to the uncertainty in the estimate of the fast ion distribution function and are taken into account. According to the study, this system is expected to be able to resolve the fast ion distribution in ten or more nodes with a temporal resolution of less than $5 \mathrm{~ms}$ for various scattering geometries. For a given input power, increasing the integration time will improve the resolving power (CTS signal). Presently, the planned integration time will be typically $2 \mathrm{~ms}$ gyrotron on time at $50 \%$ duty cycle hence a 4 ms resolution.

\section{Refraction: Spatial resolution and robustness}

The radial spatial resolution (across the flux surfaces) of CTS-AUG is between 2 and $10 \mathrm{~cm}$, depending on the scat- tering geometry. The spatial resolution is determined by the radial extend of the scattering volume. Hence in a near perpendicular scattering geometry [Fig. 2(a)], a scattering volume on the low field side (larger angle between probe and receiver beam) will have a better resolution of about $2 \mathrm{~cm}$ as opposed to an elongated scattering volumes when the scattering volume is located on the high field side (smaller angles between probe and receiver beam). Near parallel scattering geometry will have a better radial spatial resolution since the elongated axis of the scattering volume is at an angle to the flux surfaces. Additionally, it is important to investigate the robustness to refraction that varies due to sudden changes in density (such as from sawteeth activity) and due to dispersion at the spectral limits of the fast ion feature. There are three criteria for robustness to refraction effects; first, the location of the scattering volume where the measurement is taken, second, the beam overlap which directly affects the resolving power, and finally the dispersion effects within the spectral half width. Choosing $O$-mode has the advantage of being less susceptible to refraction due to its higher cutoff density than its $X$-mode counterpart. The feasibility studies reported in Ref. 15 have concluded that the density effects are marginal even for density excursions of up to $30 \%$ at very high densities of $10^{20} \mathrm{~m}^{-3}$.

\section{COMMISSIONING ACTIVITIES}

\section{A. Gyrotron frequency dynamics}

In a microwave based CTS system, the gyrotron frequency must be known to within $10 \mathrm{MHz}$. In addition, it is important to study the temporal behavior of the frequency for different gyrotron operating scenarios. This will define the 


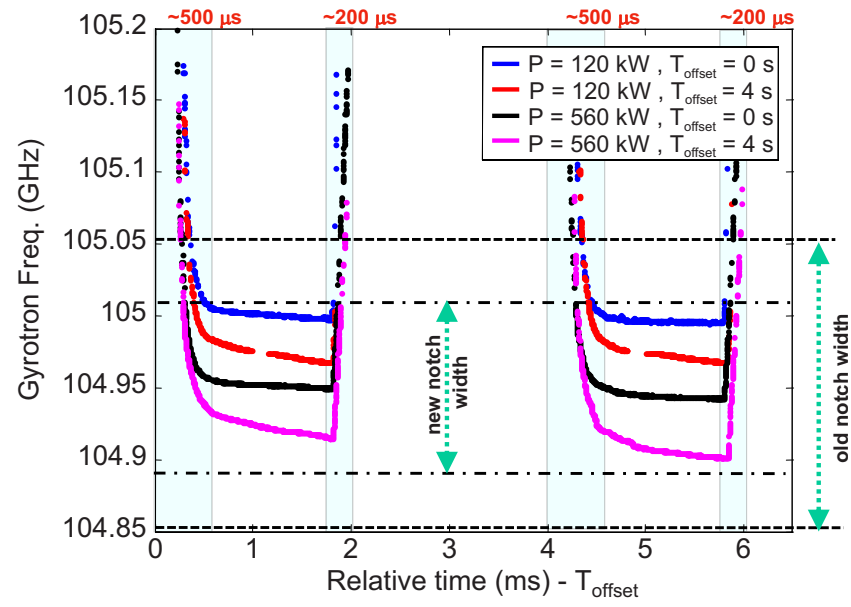

FIG. 3. (Color online) Time evolution of the Odyssey-2 gyrotron for two output powers at time $=0$ and $4 \mathrm{~s}$ of the pulse. Two pulses are shown during an on/off modulation operation with $2 \mathrm{~ms}$ on time at $50 \%$ duty cycle. The horizontal dashed line and dash-dot line are the width of the old and new notch filter width, respectively.

notch filter bandwidth and its center frequency. Detailed measurements of the frequency behavior for various gyrotron operating scenarios have been carried out. For these measurements the gyrotron power was launched into the high energy load and stray radiation was collected by a pickup horn inside the MOU box. The signal was heterodyned in a harmonic mixer driven by a stable $( \pm 0.5 \mathrm{MHz})$ sapphire tuned INVAR cavity Gunn oscillator ${ }^{16}$ with an operating range of $12.7-13 \mathrm{GHz}$. The IF signal was fed to a Tektronix Digital Phosphor Oscilloscope (model DPO 7104) with a fast 20 Mbyte frame grabbing capability digitizing at $1 \mathrm{GHz}$ bandwidth. The data were then transferred to a PC for spectrogram analysis. The frequency results plotted in Fig. 3 show the frequency range for different output powers and time. As expected, the frequency decreases with increasing power (higher electron beam voltage) due to the higher relativistic electron parallel velocity. The interesting observation here is the frequency dynamics during the rise and fall of the beam voltage which chirps by about $300 \mathrm{MHz}$-well outside the notch filter region. The frequency evolution can only be fitted using only three exponential functions each corresponding to a different time constant, namely, $\tau_{\text {thermal }}$ $>\tau_{\text {charge }}>\tau_{V}$ where the time constant $\tau_{\text {thermal }}$ is related to the thermal expansion of the cavity, $\tau_{\text {charge }}$ is the time constant related to the space charge buildup, ${ }^{97}$ and $\tau_{V}$ is the time constant related to the beam voltage rise time in the gyrotron. These measurements enable us to determine the timing for switching in the VCVA attenuation to protect the receiver from radiation with frequencies outside the rejection band of notch during the ramping phases. According to the results in Fig. 3, the VCVA should attenuate the power during the first $500 \mu$ s and the last $200 \mu$ s of the gyropulse. The frequency values during the "flattop" phase drift about $100 \mathrm{MHz}$ and are within the notch.

\section{B. Notch filters}

The notch filters used in the CTS-AUG is essentially composed of a waveguide operating in fundamental mode coupled to a number of resonators. The bandpass frequency and its width is adjusted by changing the resonator length via tuning screws. The notch depth will be dependent on the stop bandwidth and the total number of resonators. Recently, the original 22 resonator notch filters have been replaced by a redesigned 16 resonator version to improve the insertion loss over a broader bandwidth outside the filter rejection band. As a result, the insertion loss for each filter is an impressive $<2 \mathrm{~dB}$ over a broader frequency range outside the notch ranging between 98 and $107 \mathrm{GHz}$. The original filters had a depth of about $60 \mathrm{~dB}$ and $200 \mathrm{MHz}$ bandwidth (shown by the two horizontal dashed lines in Fig. 3). Stray radiation experiments on the Odyssey-2 gyrotron have concluded that the total attenuation should be $>100 \mathrm{~dB}$ to avoid gain compression of the IF amplifiers and to avoid potential damage to the mixer. In order to achieve this attenuation $(>50 \mathrm{~dB}$ each) with fewer resonators, the rejection bandwidth had to be set to a narrower $130 \mathrm{MHz}$ width. The narrower bandwidth is still well within the frequency range of the gyrotron shown as dot-dash horizontal lines in Fig. 3. If desired, an even narrower notch bandwidth will enable having more information on the bulk ion feature. However, it will limit the gyrotron operating scenario possible for CTS experiments.

\section{Spurious signals in the receiver}

It is essential that the radiation is free of addition spurious signal at other frequencies that exist outside the notch filter bandwidth. There are two fundamental types of spurious signal; one originating from the gyrotron and the other from the plasma. The spurious signal from the gyrotron can come from either other resonator modes or parasitic oscillation within the tube. The CTS receiver is very sensitive for detecting scattered radiation levels as low as $5 \mathrm{eV}$ thus spurious noise, if present, will corrupt the CTS measurements. Occurrences of gyrotron spurious signals cannot be detected by conventional directional couplers since the main gyrotron radiation can be as much as $120 \mathrm{~dB}$ higher in intensity. Experiments to investigate the gyrotron spectral purity have been carried out using the CTS receiver measuring stray radiation from modulated gyrotron (on/off $=2 / 2 \mathrm{~ms}$ ) launching power into the main transmission line load. An example is shown in Fig. 4 with two contours corresponding to different gyrotron powers; with a spurious-free spectrum (a) and a spectrum with spurious signal (b). The abscissa and ordinate denote the receiver channel number and the time, respectively. The contour values are the signal voltage normalized to the background value before the start of the gyrotron. Hence lighter (darker) color denotes detected signal (gain compression). The main gyrotron (attenuated by about $120 \mathrm{~dB}$ by the tuned notch filter) can be seen chirping between center channels 28 and 27 during every on time. During the experiments, the gyrotron power was increased incrementally until spurious signals appeared at a gyrotron power threshold of about $480 \mathrm{~kW}$. In this experiment, the frequencies of these spurious signals are arbitrary and do not correspond to possible discrete modes in the Odyssey-2 gyrotron. The cause of these spurious signal is somewhat still not fully understood. The CTS installed on the FTU tokamak which runs at below fundamental have also seen similar phenomena 


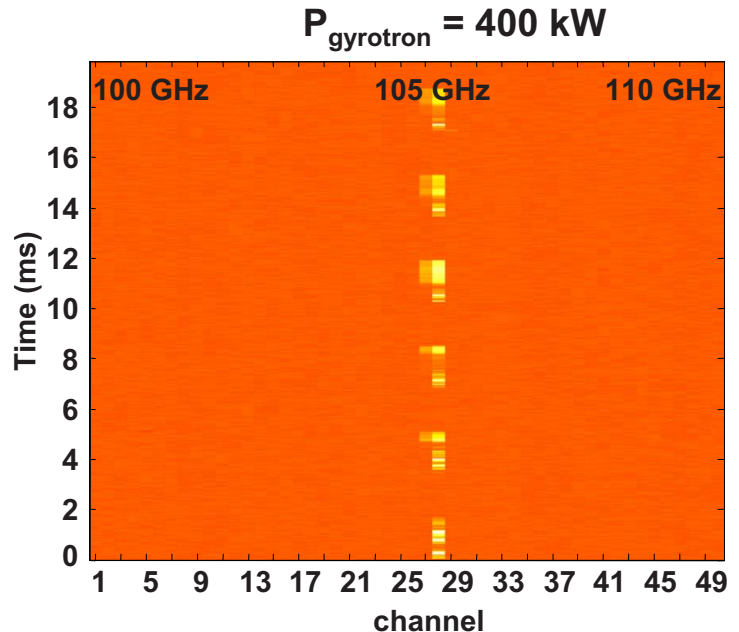

(a)

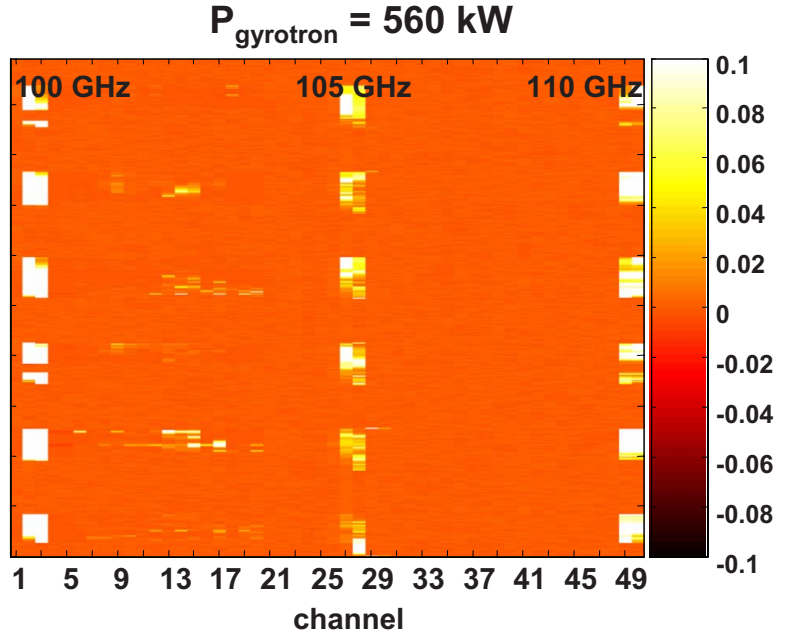

(b)

FIG. 4. (Color online) Examples of two scenarios of pure and impure spectra. Contours of measurements by the CTS receiver of the stray radiation of Odessey-2 gyrotron launched into the high power load at two different powers: (a) $400 \mathrm{~kW}$ and (b) $560 \mathrm{~kW}$. The gyrotron was modulated with 2 ms on time at 50\% duty cycle. The ordinate and abscissa axes represent the time and the frequency channels (100.0-110.0 GHz), respectively. The contour values are the voltage normalized to the initial background levels where lighter colors indicate detected signal and dark color denotes gain compression. Main gyrotron radiation is detected by the center channel (Nos. 28-27) which are deep in the notch.

where they refer it to "anomalous spectra" and concluded that is was caused by backreflections from breakdown plasma or arcs in the transmission line. ${ }^{18}$ There are currently three hypothesis: the first is that spurious signals intrinsically come from the gyrotron for specific gyrotron parameter settings (beam voltage, coil current, etc.) where the conversion efficiency it not optimized and cause parasitic oscillations. The second hypothesis is backreflected radiation from either a surface or from arcs in the transmission line that affects the electron beam in the resonator causing spurious signal. The third hypothesis is a combination of the two where certain gyrotron settings may make the gyrotron more (or less) robust to backward reflected radiation. Each of these hypothesis can explain the experimental observations whereby gyrotron spurious signal appearing with larger gyrotron power. The first hypothesis explanation: the gyrotron output power in these experiments was achieved by only increasing the beam voltage, thus affecting electron pitch angle in the beam, changing the efficiency, causing spurious signal. Second hypothesis explanation: increasing the gyrotron power will increase the probability of arcs thus reflecting back more radiation. Third hypothesis explanation: increasing the gyrotron power increases the probability of arcs and thus reflected power. In addition, by only increasing the beam voltage decreases the robustness to backreflections of radiation. Further studies are needed to fully explain these phenomena. At any rate, the stray radiation experiments have put an upper limit of the gyrotron power of $450 \mathrm{~kW}$ at the current gyrotron settings. However, CTS experiments have demonstrated that some spurious signal can also originate from the plasma rather than the gyrotron even for lower gyrotron power of $200 \mathrm{~kW}$ and for certain experimental conditions. It is still not clear of the cause of these plasma generated spurious signal. One plasma scenario that eliminated the plasma generated spurious signals was operating in the lower confinement mode ( $L$-mode) and moving the plasma inward away from the receiver first mirror. The commissioning phase to study and eliminate the plasma generated spurious signal in the standard high confinement ( $H$-mode) plasma scenario is in progress.

\section{Ex-vessel transmission line}

Careful design, construction, alignment, and quality assurance of the of the CTS transmission section and its coupling to the ECRH transmission line are important not only to achieve low loss transmission but also to provide good spatial localization of the scattering volume. The first phase of the alignment process is achieved using a diode laser. To measure the beam pattern at different locations in the transmission, a compact two-dimensional scanning rig (microrig) equipped with a sniffer probe connected to $110 \mathrm{GHz}$ detector diode has been constructed at Ris $\varnothing$-DTU. This valuable tool enables us to not only to improve the alignment but also verify the beam quality. A $110 \mathrm{GHz}$ Gunn oscillator or a backward wave oscillator is installed in the fundamental waveguide portion of the receiver to transmit a signal outward through the receiver horn into the transmission line in the reverse direction. It is important that emitted power from the rf source be well below the nonlinear portion of the diode detector. Measurements of the beam pattern in the transmission line has shown misalignment and astigmatism and clearly indicates that the laser alignment for the quasioptical components is insufficient. The challenge was to not only to align and couple the CTS mirrors to the ECRH free space propagation section of the transmission line but also to avoid astigmatism. Hence, a technique was developed using a twoway laser and the microrig where each beam segment is aligned. A HE11 coupler ${ }^{19}$ was constructed that transforms a Gaussian beam from a horn to the HE11 mode. The HE11 coupler was inserted in one of the Mitre bends and radiation propagates toward the MOU box. Starting from the wave- 

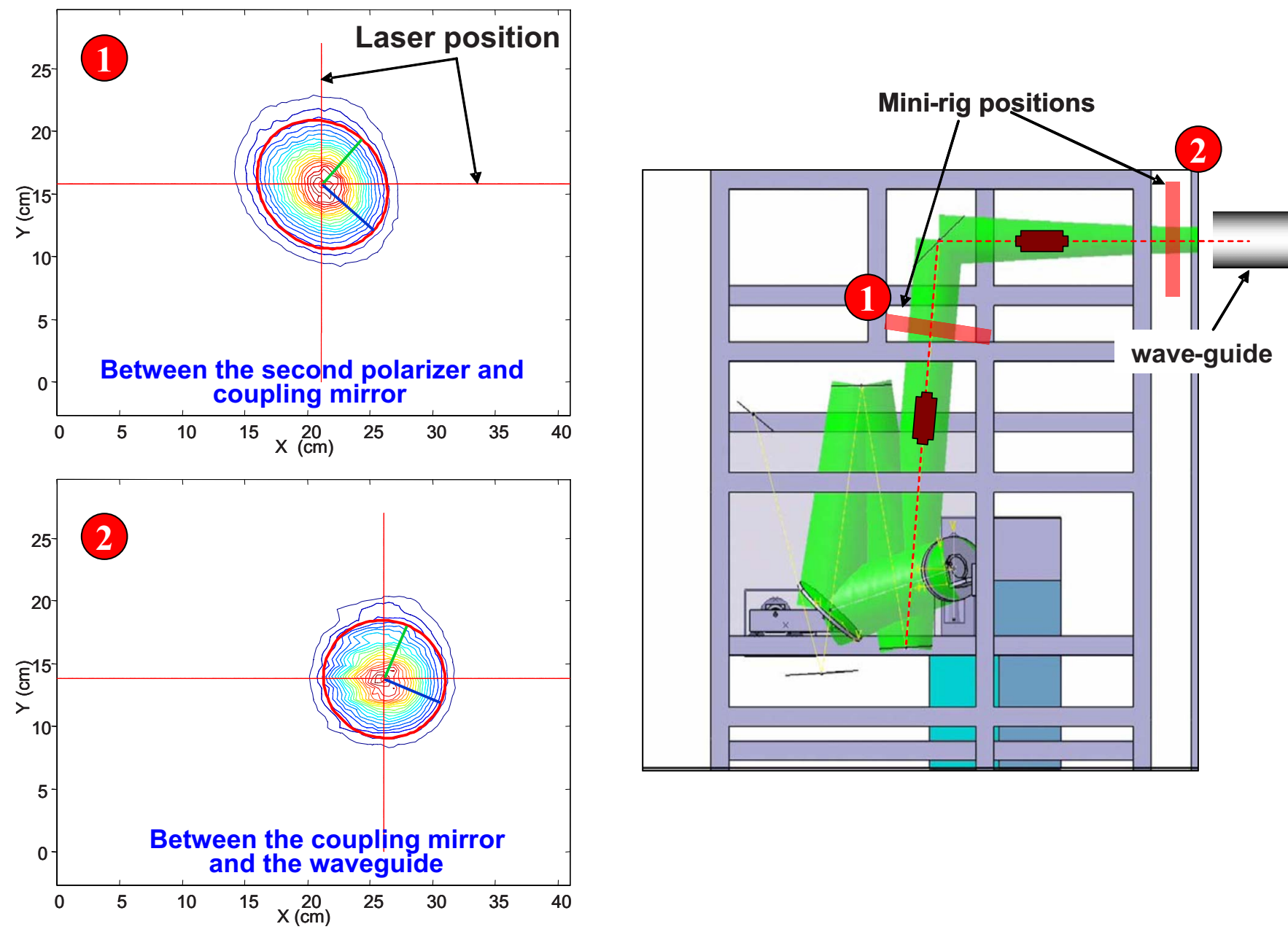

FIG. 5. (Color online) The piecewise alignment procedure. The left two graphs show the beam pattern measured at the corresponding sections in the ECRH MOU box transmission line shown on the right. The cross hairs are the laser reference position from the two-way laser that connects the geometrical centers of two mirrors.

guide to the horn entrance, the piecewise alignment is carried out between two components. The two-way laser is used as a reference that connects the geometrical centers of the two components. The laser spot is referenced by the microrig position. The laser is then removed and the microrig measures the beam pattern in between. The mirror angles of the first of the two components are adjusted to match the beam pattern maximum to the laser reference. Figure 5 (right) shows the setup and two examples of alignment sections; between the second polarizer and the coupling mirror (No. 1), and between the coupling mirror and the wave guide (No. 2). To the left of Fig. 5 are the beam pattern measurements after the mirrors have been modified to match the laser reference marked by red crosses on the figures. This alignment procedure does not include the angle of the horn with respect to the mirror. Thus, the final stage of the alignment process carried out by reversing the radiation out through the horn toward the waveguide. The microrig measures the beam pattern at the entrance of the wave guide. The two-way laser references the microrig to the geometrical center of the waveguide The angle of the horn is then slightly adjusted while keeping the center position fixed until the measured beam is centered on the waveguide hence matching the two alignments. The measured beam centers at each section of the quasi optical transmission line agree very well with the Gaussian beam calculations. The transmission throughput of the entire transmission (except the in-vessel section) was improved to about $85 \%-90 \%$ which is close to the expected theoretical estimates, taking into account the losses in the Mitre bends in $70 \mathrm{~m}$ of wave guide transmission line. The beam pattern measured at the end of the waveguide transmission line in the torus hall was nearly $95 \%$ circular with no side lobes. Tests have shown that the most sensitive part of the MOU transmission line is the receiver horn position and the mirror coupling the beam to the waveguide shown in Fig. 5.

\section{E. In situ beam measurements inside the vacuum vessel}

The microrig was used to measure beam pattern inside the vacuum vessel. In addition for beam quality assurance, these measurements were also performed to verify the alignment. The ECRH group performed laser calibration to create a transformation map between antenna settings and angles (poloidal and toroidal). To compare the receiver laser alignment to the microwave beam pattern have been measured for different antenna settings. First, the measured beam in the 


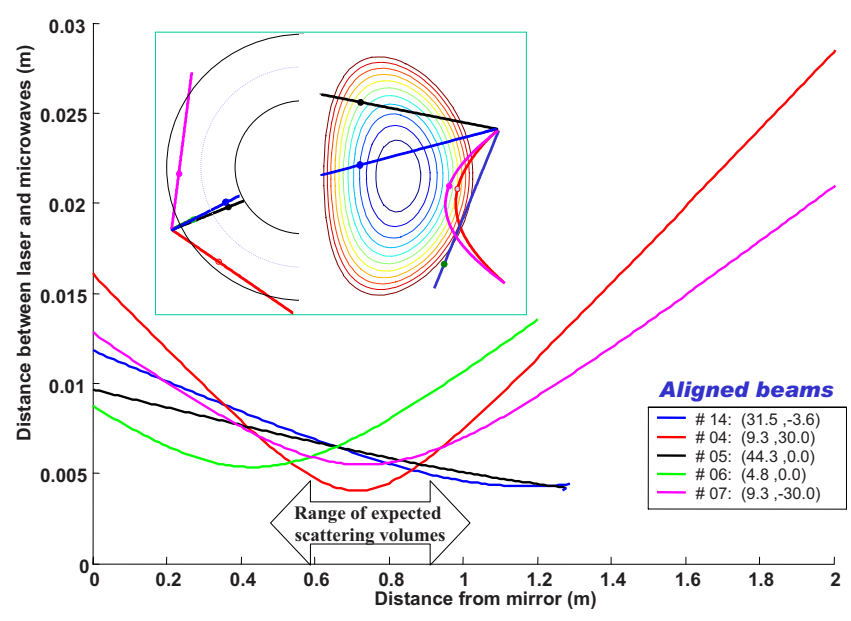

FIG. 6. (Color online) The distance between the measured laser and the microwave line as a function of distance from the steerable mirror measured inside the ASDEX Upgrade vacuum vessel. The line colors correspond to different antenna settings depicted by the top view and poloidal view in the insets.

vacuum vessel shows no sidelobes for all extreme antenna angles settings to the within the sensitivity of the diode. The beam widths also agree well with Gaussian calculations. The microwave beam vector was determined by spatial measurements of the microrig waveguide located at the maximum beam radiation. A high precision calibrated arm (FARO arm), supplied and operated by the ASDEX Upgrade staff, measured the global coordinate position at two distances from the steerable antenna essentially creating a three-dimensional $3 \mathrm{D}$ vector. A direct comparison of the distance between the microwave and laser line as a function of the distance from the steerable mirror is shown in Fig. 6 for five extreme antenna angle settings. The top and poloidal views of the antenna settings are shown in the graph inset. The spatial error bar of $5 \mathrm{~mm}$ was determined from the fundamental wave guide dimensions, the dimensions of the FARO arm point, and by repeating the measurements. These results show that the difference between the microwaves and the laser on the first mirror is between 1 and $1.5 \mathrm{~cm}$. The distance between the in-vessel waveguide and the antenna is $0.978 \mathrm{~m}$. The angle between the laser and microwave beam between the waveguide and the steerable mirror is difficult to assess since we do not know the position of the exiting beam with respect to the waveguide center and the errors in question are considerable. However, it is reasonable to conclude that the angle is below $1^{\circ}$. Figure 6 shows that the beam and laser converge toward each other. Thus we can conclude that the scattering volumes for all scattering geometries have an uncertainty between the laser and microwave beam less than 1 $\mathrm{cm}$. The overlap experiments in Sec. V show that these measurements are consistent with a well aligned system.

\section{F. Measurement and characterization of the ECRH polarizers for the CTS-AUG}

The choice of $O$-mode radiation is achieved using ECRH broadband polarizers. A coherent polarized source was launched from within the vacuum vessel and the polarization properties (ellipticity, angle of ellipticity, and phase

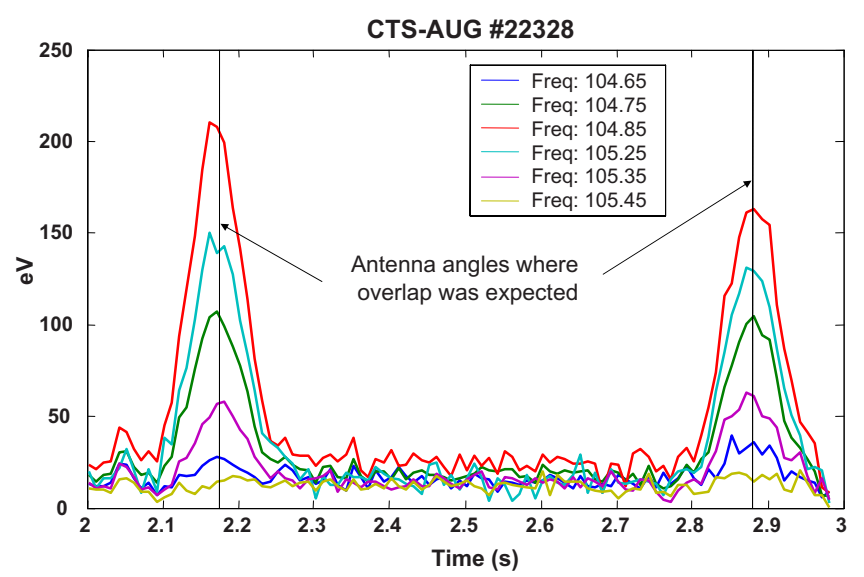

FIG. 7. (Color online) Time traces of the scattered radiation for the centermost channels during a double poloidal scan of the receiver beam across the probe beam. The discharge was Ohmic and $\angle\left(k^{\delta}, B\right) \approx 100^{\circ}$. The two vertical lines are the time points where the receiver antenna position is expected to have maximum overlap from calculations based on prior in-vessel alignment of the antenna and ray tracing. The density during the second poloidal sweep was decreased for comparison.

direction) as a function of polarizer settings for the CTS mirrors in the MOU box has been measured at the end of the transmission line. This was performed using a device constructed at Ris $\varnothing$ consisting of an orthogonal pair of fundamental waveguide detectors. The results were compared to polarizer codes from both IPP and Ris $\varnothing$ and they all agree to within the measurement error bars. More details of the device and the technique can be found in Ref. 20.

\section{PRELIMINARY SCATTERING RESULTS}

Using the plasma scenario that remedied the spurious signal (mention in Sec. IV C), preliminary CTS scattering experiments were carried out on ASDEX Upgrade. One such experiment was an overlap scan in near perpendicular $\angle\left(k^{\delta}, B\right) \approx 100^{\circ}$ scattering geometry performed in Ohmic discharges. The gyrotron beam was modulated with on/off time of $2 / 2 \mathrm{~ms}$. The CTS signal is the difference between the signal and the fit during the gyroperiods. Figure 7 shows the time traces of the CTS signal of several inner channels closer to the gyrotron frequency during two sweeps of the receiver antenna across the probe beam during the discharge. The vertical lines are the time points where the receiver antenna position is expected to have maximum overlap from calculations based on prior in-vessel alignment of the antenna and ray tracing. There is good agreement. The same experiments were performed for near parallel scattering geometry $\angle\left(k^{\delta}, B\right) \approx 150^{\circ}$ and the results also show very good agreement of the alignment between experiment and calculation. The scattering geometries for both experiments are shown in Fig. 2 where the beams were oriented toward the counterion flow direction. Thus as expected, the results show the frequency upshift due to the fast ion flow direction in NBI heated plasmas. The first scattered spectra for near perpendicular scattering geometry in an AUG ICRH heated plasma (minority hydrogen, $R_{2 \omega \mathrm{H}} \approx$ center) are shown in Fig. 8. The frequency range shown is between 105.4 and $106.5 \mathrm{GHz}$ (gyrotron frequency $=104.95 \mathrm{GHz}$ ) which corresponds to 


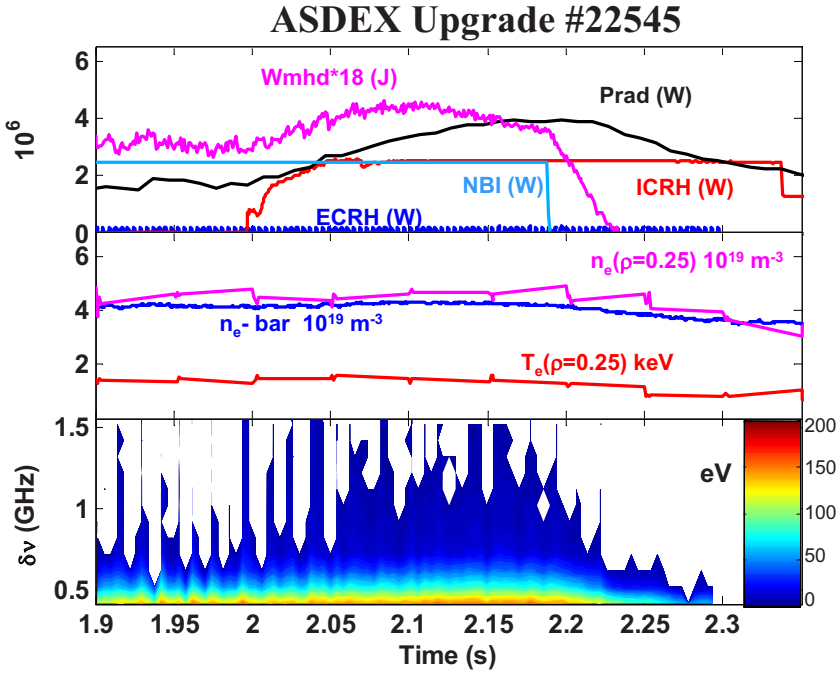

FIG. 8. (Color online) Time traces of the ICRH and neutral beam heated ASDEX Upgrade $L$-mode discharge. The top graph shows the traces of the ECRH, ICRH, total radiated power, NBI, and the stored energy from MHD scaled by 18 . The middle graph shows the time trace of the core line integrated density from the interferometer and the density and temperature at $\rho=0.25$ from the Thomson scattering. The bottom graph is the contour of the CTS scattered spectra for $\angle(k \delta, B) \approx 100^{\circ}$ and scattering volume located at the plasma center. The high frequency portion of the scattered spectra is plotted $(+\delta v 0.4$ and $1.5 \mathrm{GHz})$ corresponding to the energy range for hydrogen of $2-20 \mathrm{keV}$.

$\sim 2-20 \mathrm{keV}$ for hydrogen. The plasma scenario for coupling ICRH power is still not optimized. However, the increasing Wmhd during the ICRH ramp-up phase suggests that some heating is occurring until it decreases again most probably due to the increase in impurities indicated by the Prad increase. The CTS scattered spectra in Fig. 8 broaden during the ICRH ramp-up while the density and temperature remain nearly constant. Impurities such as tungsten and carbon, which are the main contributors to the Prad, can distort the spectra and can explain the broadening. However, scattering simulations have shown that due to their higher mass, this should only occur for the portion of the spectra below 0.4 $\mathrm{GHz}$ from the main gyrotron frequency. Therefore the scattering at higher frequencies region suggests some indication of hydrogen heating from ICRH.

\section{DISCUSSION AND COMMISSIONING ACTIVITIES TO BE COMPLETED}

This paper has covered the commissioning activities highlighting the prerequisites and pitfalls of a microwave based CTS system. The piecewise alignment technique was successful in coupling the CTS mirrors to the ECRH transmission line portion in the MOU. This not only improved the alignment but also significantly reduced the astigmatism in the beam. The in-vessel measurements and the overlap experiments have concluded that the CTS-AUG is well aligned and the absolute spatial uncertainty of the scattering volume in the plasma is to within $1 \mathrm{~cm}$. The key pitfalls are spurious signals that exist in the receiver frequency bandwidth that hamper the experiments. The source of the spurious signal can come from either the gyrotron and/or plasma. At present, a plasma generated spurious-free operating scenario thus far exists during $L$-mode operation with plasma edge moved away from the receiver antenna. Preliminary scattering experiments have shown encouraging results of some evidence of ICRH heating. However, analysis and elimination of the spurious signals in standard operating scenario are in progress on ASDEX Upgrade. The final stage of the commissioning will be the analysis of the scattering signal strength versus plasma background to infer the fast ion distribution from the measured scattered spectra. Experience learned from the CTS-AUG is a stepping stone to a CTS system on larger devices such as ITER. Although technically demanding, CTS can be a rich source of information on the fast ions and their behavior.

\section{ACKNOWLEDGMENTS}

The authors wish to thank the ASDEX Upgrade team for their support in the experiments that made the preliminary CTS results possible. In addition, we would like to thank the technicians from Ris $\varnothing$-DTU and the Asdex Upgrade ECRH group for their support. Lastly, we are also grateful to Max Münich for discussions on microwave experiments.

${ }^{1}$ E. E. Salpeter, Phys. Rev. 120, 1528 (1960).

${ }^{2}$ P. Woskoboinikow, Rev. Sci. Instrum. 57, 2113 (1986).

${ }^{3}$ H. Bindslev, J. Atmos. Terr. Phys. 58, 983 (1996).

${ }^{4}$ R. Aamodt and D. Russell, Nucl. Fusion 32, 745 (1992).

${ }^{5}$ H. Bindslev, J. Plasma Fusion Res. 76, 878 (2000).

${ }^{6}$ H. Bindslev, J. A. Hoekzema, J. Egedal, J. A. Fessey, T. P. Hughes, and J. S. Machuzak, Phys. Rev. Lett. 83, 3206 (1999).

${ }^{7}$ S. K. Nielsen, H. Bindslev, L. Porte, J. A. Hoekzema, S. B. Korsholm, F. Leipold, F. Meo, P. K. Michelsen, S. Michelsen, J. W. Oesterbeek et al., Phys. Rev. E 70, 016407 (2007).

${ }^{8}$ H. Bindslev, S. K. Nielsen, L. Porte, J. A. Hoekzema, S. B. Korsholm, F. Meo, P. K. Michelsen, S. Michelsen, J. W. Oesterbeek, E. L. Tsakadze et al., Phys. Rev. Lett. 97, 205005 (2006).

${ }^{9}$ D. Wagner, G. Grünwald, F. Leuterer, A. Manini, F. Monaco, M. J. Münich, H. Schütz, J. Stober, H. Zohm, T. Franke et al., IEEE Trans. Plasma Sci. 36, 324 (2008).

${ }^{10}$ S. Michelsen, S. B. Korsholm, H. Bindslev, F. Meo, P. K. Michelsen, E. L. Tsakadze, J. Egedal, P. Woskov, J. A. Hoekzema, F. Leuterer et al., Rev. Sci. Instrum. 75, 3634 (2004)

${ }^{11}$ S. B. Korsholm, H. Bindslev, F. Meo, F. Leipold, P. K. Michelsen, S. Michelsen, S. K. Nielsen, E. L. Tsakadze, P. Woskov, E. Westerhof et al., Rev. Sci. Instrum. 77, 10E514 (2006).

${ }^{12}$ G. G. Denisov, A. G. Litvak, V. E. Myasnikov, and E. M. Tai, Proceedings of the Sixth International Vacuum Electronics Conference, IVEC 2005, Noordwijk, The Netherlands, April 2005, Paper No. WPP-246, pp. 497-500.

${ }^{13}$ C. Mueller of General Atomics, P.O. Box 85608, San Diego, CA 92186-5608.

${ }^{14}$ H. Binslev, JET Report No. JET-R(97)13, 1997.

${ }^{15} \mathrm{H}$. Bindslev, Application for Preferential Support, Fast Ion Collective Thomson Scattering diagnostic at ASDEX Upgrade, January 2002.

${ }^{16}$ Constructed by M. Münich of the ECRH group at Max Planck Institute für Plasma Physik

${ }^{17}$ A. W. Fliflet, R. C. Lee, S. H. Gold, W. M. Manheimer, and E. Ottt, Phys. Rev. A 43, 6166 (1991).

${ }^{18}$ U. Tartari, G. Grosso, G. Granucci, F. Gandini, S. Garavaglia, G. Grossetti, A. Simonetto, V. Mellera, V. Muzzini, L. Lubyako et al., Rev. Sci. Instrum. 78, 043506 (2007).

${ }^{19}$ Designed by W. Kasparek at Institut für Plasmaforschung, Pfaffenwaldring 31, 70569 Stuttgart, Germany.

${ }^{20} \mathrm{~F}$. Leipold, S. K. Nielsen, and F. Meo, in Proceedings of the 35th IEEE International Conference on Plasma Science (ICOPS 2008), Karlsruhe, Germany, 2008. 